Muro de la Investigación, 2021(2), julio-diciembre ISSN: 2523-2886

DOI:https://doi.org/10.17162/rmi.v6i2.1638

\title{
Funcionamiento familiar y adicción a redes sociales en estudiantes del nivel secundario
}

\author{
Family functioning and addiction to social networks in secondary school students
}

\author{
Ursula Rosali Lachuma Huansi ${ }^{1 \mathrm{a}}$
}

Facultad de Ciencias de la Salud, Universidad Peruana Unión, Lima, Perú ${ }^{1}$

Recibido: 10 de diciembre de 2020

Aceptado: 15 de junio de 2021

\begin{abstract}
Resumen
La presente investigación ${ }^{\mathrm{b}}$ tuvo como objetivo estudiar la asociación que existe entre funcionamiento familiar y adicción a redes sociales en estudiantes del nivel secundario de una institución educativa pública de Lima Este. Es de diseño no experimental, de corte transversal y de alcance correlacional. La muestra estuvo conformada por 603 estudiantes, de entre 12 a 18 años de edad. Los resultados de acuerdo a la muestra de investigación analizada, revela que existe una asociación altamente significativa entre funcionamiento familiar y adicción a redes sociales. Asimismo, en relación a las dimensiones del funcionamiento familiar, se encontró asociación altamente significativa entre cohesión familiar y adicción a redes sociales $\left(\mathrm{X}^{2}=33,236 ; \mathrm{p}=, 000\right)$. De la misma forma, se halló asociación altamente significativa entre adaptabilidad familiar y adicción a redes sociales $\left(X^{2}=40,318 ; p=, 000\right)$. Cabe destacar, que en esta investigación los adolescentes con familias con un nivel de cohesión y adaptabilidad alto, mostraron menor riesgo de presentar una adicción a redes sociales.
\end{abstract}

Palabras clave: Funcionamiento familiar, adicción a redes sociales, Cohesión familiar, adaptabilidad familiar y estudiantes del nivel secundario.

\begin{abstract}
This research aims to study the association between family functioning and social media addiction in students at the secondary level of a public educational institution in the eastern part of Lima. It is non-experimental, cross-cutting and correlal-range design. The sample consists of 603 students, aged between 12 and 18. The results according to the research sample analyzed reveal that there is a highly significant association between family functioning and social media addiction. Also, in relation to the dimensions of family functioning, a highly significant association was found between family cohesion and social media addiction $\left(\mathrm{X}^{2}=33,236 ; \mathrm{p}=., 000\right)$. Similarly, a highly significant association was found between family adaptability and social media addiction $\left(X^{2}=40,318 ; p=., 000\right)$. It should be noted that in this research adolescents with families with a high level of cohesion and adaptability showed a lower risk of social media addiction.
\end{abstract}

${ }^{\mathrm{a} C}$ Correspondencia al autor:

E-mail: ursula.lachuma@upeu.edu.pe

${ }^{\text {b}}$ La presente investigación se basa en la tesis de maestría de la autora:

http://hdl.handle.net/20.500.12840/3357 
Keywords: Family functioning, social media addiction, Family cohesion, family adaptability and secondary level students.

\section{Introducción}

En la actualidad, el crecimiento y desarrollo de nuevas tecnologías han permitido descubrir otras formas de comunicación, una de ellas, las redes sociales, la cual permite tener una comunicación fluida y constante entre personas o grupos afines, asimismo, permite comunicarse con cualquier otra persona del mundo de forma instantánea sin importar dónde esta se encuentre (Urquijo, 2017). A pesar de los múltiples beneficios que brinda, las redes sociales pueden traer consigo aspectos negativos que interfieren en la vida cotidiana del individuo. Las redes sociales al ser tan dinámicas, interactivas y de fácil acceso magnetizan al usuario haciendo que este pierda la noción del tiempo, pasando muchas horas en estas y descuidando a su familia, amigos, estudios o trabajo, afectando prácticamente todos los aspectos de su vida (Sammarco, 2019; Echeburúa \& Corral, 2010).

Por su parte, Vilca y Vallejos (2015) definen la adicción a redes sociales como el deterioro de la capacidad de control, que es ocasionada directamente por una dependencia generalizada y mostrando síndrome de abstinencia al quedar privado del acceso a las redes sociales. Esta adicción trae como resultado una disminución en el rendimiento académico, modificación del estado de ánimo, dependencia, pérdida del interés por otras actividades y conflicto en el área familiar y social.

Por ello, la adicción a redes sociales es una compulsión que provoca en las personas necesidad por estar siempre conectado a la red, compartiendo fotos, vídeos o interactuando con otras personas, pasando excesivo tiempo en estas y experimentado malestar cuando no están en línea, esta adicción hace que el individuo se aísle de las demás personas, provocando ansiedad, afectando la autoestima y mermando la capacidad de control de las personas (Gavilanes, 2015; Navarro \& Rueda, 2007; Echeburúa \& Corral, 2010).

Por otro lado, la familia juega un papel muy trascendental en el desarrollo de una persona, porque es la encargada de trasmitir valores éticos y culturales a cada uno de sus miembros que les permitirá desenvolverse correctamente en el ambiente familiar y social. Asimismo, la familia es la fuente más importante de apoyo social y psicológico de una persona, ya que, tiene la función de proteger y apoyar a cada uno de sus integrantes frente a múltiples dificultades que se presenten a lo largo de su vida, razón por la cual, se convierte en el principal recurso de prevención de daños físicos y psicológicos (Suárez \& Vélez, 2018; Sigüenza, Buñay \& Guamán, 2017). Cabe destacar, que la familia es la mediadora entre el 
individuo y la sociedad, es donde se desarrolla la identidad y el proceso de socialización del sujeto, es también donde vive sus primeras experiencias, adquiere valores y su concepción del mundo, es por tal motivo, que la familia constituye para el individuo un lugar donde se dan las condiciones para el desarrollo favorable y sano de su personalidad, o, por el contrario, puede llegar a ser la fuente principal de sus trastornos psicológicos (Zaldívar, 1998) .

Olson, Sprenkle y Russell (2012) definen el funcionamiento familiar como la interacción de vínculos afectivos y emocionales que existe entre los miembros de la familia y el grado de autonomía que el individuo perciba dentro de su núcleo familiar (cohesión), y que además pueda ser capaz de modificar su estructura con la finalidad de superar dificultades o situación que se presenten a lo largo de la vida familiar (adaptabilidad). Un funcionamiento familiar saludable, permitirá desarrollar una adecuada dinámica familiar en la que existe determinadas pautas de interrelación entre los integrantes de la familia, las cuales son matizadas por los sentimientos, afectos y emociones expresados entre sí y como familia (Zaldívar, 2012).

Es así, que las redes sociales dentro del funcionamiento familiar han generado mucha polémica, debido a que los primeros años de vida son muy importantes y críticos para el desarrollo de los niños, es por esta razón, que las redes sociales pueden favorecer o distorsionar la vida familiar, ya que, al pasar tiempo en estas, los miembros pueden perder el interés por compartir tiempo o actividades como familia (Pinto, 2018).

Una adicción a redes sociales tiende a afectar a personas que psicológicamente o por la edad son más vulnerables (Echeburúa, Labrador \& Becoña, 2009). Un grupo de riesgo al que hay que prestarle mucha atención son los adolescentes, ya que esta etapa es crítica para ellos, porque se dan cambios físicos, psíquicos y sociales, además de presentar dificultad para controlar sus impulsos, vulnerabilidad ante la publicidad, baja tolerancia a la frustración, problemas de identidad, poseen poca experiencia de vida, son incapaces de reconocer adicciones sutiles y tampoco son conscientes del riesgo que trae consigo (Morales, 2013). Durante esta etapa, los adolescentes centran su vida principalmente en su grupo de amigos, pasando más tiempo con ellos y compartiendo menor tiempo con su familia, haciendo muchas veces que la relación entre padres e hijos se debilite, lo que provoca mayor vulnerabilidad frente a una adicción (Rayo, 2014). Asimismo, son los adolescentes y jóvenes del sexo masculino que tienen entre 13 a 24 años de edad quienes más acceden a las redes sociales en el Perú (We are social \& Hootsuite, 2019).

En este sentido, Aponte, Castillo y Gonzales (2017) realizaron una investigación sobre prevalencia de adicción a internet y disfunción familiar en 390 adolescentes ecuatorianos de 15 a 19 años de edad, encontrando que los adolescentes que presentaban 
adicción a la internet provenían de familias disfuncionales en la que había bajos niveles de cohesión familiar, afectividad y comunicación. Asimismo, Aguirre y Zurita (2015) realizaron una investigación sobre funcionamiento familiar y ciberadicción en 581 adolescentes ecuatorianos de 11 a 20 años, encontrando que el 51, $8 \%$ de adolescentes que presentaban adicción a redes sociales provenían de familias disfuncionales, lo que significa que, la disfunción familiar es un factor de riesgo para las adicciones a redes.

En este sentido, Maldonado (2015) realizó una investigación con la finalidad de estudiar la relación entre las redes sociales y la convivencia familiar, participaron 126 adolescentes ecuatorianos de 11 a 19, los resultados indicaron que existe una relación positiva, es decir, las redes sociales influyen de forma negativa en la convivencia familiar, dado que, a mayor uso de redes sociales, menor tiempo de convivencia familiar. Por otro lado, Ángel y Álzate (2015) efectuaron una investigación sobre relaciones sociales y familiares en 114 adolescentes colombianos usuarios de redes sociales, con edades de 10 a 17 años, los resultados encontrados revelan que no hay tendencias significativas a nivel estadístico que considere negativo el uso de redes sociales.

Una investigación realizada en Perú sobre el abuso de las nuevas tecnologías ubicó a este como uno de los países donde más se accede a redes sociales, haciendo que se le considere como una fuente de riesgo, teniendo en cuenta que, a partir de ello, pueden desencadenarse trastornos de conductas relacionadas con el abuso de redes sociales (Vallejos \& Capa, 2010). Otra investigación realizada en Perú por Cruzado, Matos y Kendall (2006) reveló que el $80 \%$ de pacientes que presentaban adicción a redes sociales, mostraban un amplio historial de disfunción familiar, evidenciado falta de soporte por parte de su familia, lo que indica que es uno de los factores determinantes que anteceden a una adición a redes sociales. Por otro lado, Zapata (2013) realizó una investigación en el Hospital Hermilio Valdizán en Perú con 186 adolescentes de 14 y 15 años de edad que fueron atendidos en consulta externa, encontrando que el 57, $4 \%$ proviene de familias disfuncionales.

Por su parte, Córdova y Dávila (2017) realizaron una investigación sobre clima social familiar y adicción a redes sociales con 124 estudiantes peruanos de 13 a 19 años de edad, encontrando relación significativa entre las variables, es decir que, a menor clima social familiar, mayor probabilidad de que un adolescente presente riesgo de adicción a redes sociales, ya que no existe comunicación, apoyo, ni control por parte de la familia. De igual manera, Adriano y Mamani (2015) efectuaron una investigación sobre la relación entre el funcionamiento familiar y adicción a internet en 225 adolescentes peruanos de entre 10 a 24 años, encontrando que la mayoría de adolescentes mostraban un uso normal del internet, sin embargo, los adolescentes que provenían de familias con funcionamiento familiar extremo, 
tenían mayor probabilidad de presentar una adicción a internet. Esto significa que, las personas con familias disfuncionales presentan el doble de riesgo de padecer una adicción a redes sociales, en comparación con aquellos que experimentan ambientes adecuados de crianza y que, por lo tanto, se sienten escuchados, amados y apoyados (Vallejos \& Capa, 2010).

Desde otro ángulo, esta investigación permitirá a las diferentes autoridades tomar medidas pertinentes para la implementación de programas de intervención, charlas de sensibilización y talleres educativos para así lograr el fortalecimiento familiar y el uso adecuado de redes sociales. Así como también, estará disponible para cualquier persona que esté interesada en conocer más en profundidad el rol que juega la familia frente a una adicción a redes sociales en adolescentes. Por otro lado, se pretende realizar la validez de contenido y confiabilidad de los instrumentos aplicados para que se conviertan en instrumentos cotidianos para otros investigadores que estén interesados en trabajar con variables y población similar con el propósito de generalizar los resultados encontrados. Finalmente, esta investigación tiene como objetivo determinar si existe asociación significativa entre funcionamiento familiar y adicción a redes sociales en estudiantes del nivel secundario de una institución educativa de Lima Este.

\section{Metodología}

Esta investigación posee un enfoque cuantitativo porque se basó en la recolección y análisis de datos, es de diseño no experimental porque las variables no fueron controladas ni manipuladas, ya que el propósito fue observarlas en su contexto natural, para posteriormente analizarlas (Sánchez, Reyes \& Mejía, 2018). Es de corte transversal porque el proceso de recolección de datos se dio en un momento determinado, finalmente, es de tipo correlacional porque se busca encontrar el grado de asociación entre funcionamiento familiar y adicción a redes sociales (Hernández, Fernández \& Baptista, 2005).

\section{Participantes}

El grupo de participante estuvo conformado por 603 estudiantes del nivel secundario de una institución educativa pública de Lima este. Toda la población fue del sexo masculino y tenían edades entre 12 y 18 años, los cuales estaban matriculados en el año escolar y contaban con el consentimiento informado firmado por sus padres o apoderado. El tipo de muestreo fue no probabilístico, a conveniencia de la investigadora. 


\section{Instrumentos}

El instrumento utilizado para la recolección de datos del funcionamiento familiar fue la Escala de Cohesión y Adaptabilidad Familiar (FACES III), elaborado por Olson, Portner y Lavee en Estados Unidos (1985). Este instrumento evalúa el funcionamiento familiar en base a sus dos dimensiones: cohesión y adaptabilidad familiar, la administración puede ser de forma individual o colectiva en sujetos de 12 años en adelante, con una duración de 15 minutos aproximadamente. La escala cuenta con 20 ítems: 10 para cohesión familiar y 10 para adaptabilidad familiar, que deberán ser puntuada a través de una escala Likert, con un rango de uno a cinco puntos respectivamente: nunca, casi nunca, algunas veces, casi siempre y siempre. La adaptación del FACES III en el Perú fue realizada por Reusche Lari (1994), a través del criterio de jueces. El análisis de confiabilidad se realizó mediante el coeficiente Alpha de Cronbach para cada una de las dimensiones: cohesión (0.78) y adaptabilidad (0.56). En cuanto a la validez del constructo se efectuó el análisis correlacional $r$ de Pearson ítems subtest. Los resultados muestran que las correlaciones oscilan entre 0.287 a 0.714 (Zambrano, 2011; Adriano \& Mamani, 2015). Cabe destacar que en esta investigación se utilizó la norma actualizada del FACES III, habiendo cambios radicales en las interpretaciones “altos puntajes en las escalas de cohesión y adaptabilidad son indicativos de sistemas balanceados, mientras que, puntajes bajos en las mismas escalas son reflejo de sistemas desbalanceados" (Bazo, et al., 2017).

Por otro lado, el instrumento utilizado para medir adicción a redes sociales fue el Cuestionario de Riesgo de Adicción a Redes Sociales (CrARS), elaborado por los psicólogos peruanos Vilca y González (2013). Este cuestionario cuenta con 43 ítems y 7 dimensiones que son: pérdida del control, síndrome de abstinencia, disminución del rendimiento académico, modificación del ánimo, dependencia, pérdida del interés por otras actividades e interferencia en el área social. Puede ser aplicada de manera individual o colectiva en adolescentes de 14 a 18 años, teniendo una duración de 15 minutos aproximadamente. El constructo cuenta con opciones de respuesta tipo Likert: nunca, casi nunca, algunas veces, muchas veces y siempre; con puntuaciones que van desde 1 a 5 respectivamente (Vilca \& González, 2013). La adaptación a una población de adolescentes de 11 a 18 años, lo realizó el psicólogo Carlos Castro en el año 2018, a partir del criterio de jueces. En cuanto a la fiabilidad, el instrumento muestra un Alpha de Cronbach de 0.92 para la escala general y para sus dimensiones un puntaje que oscila entre los 0.62 y 0.71 respectivamente, mostrando que el instrumento es fiable. Para determinar la validez de contenido se utilizó la V de Aiken evidenciando valores entre 0.8 y 1.00 respectivamente, lo que indica que el instrumento puede ser utilizado sin necesidad de modificar algún ítem (Castro, 2019). 


\section{Análisis de datos}

Para el análisis estadistico se usó el paquete estadístico IBM SPSS Statistics para Windows Versión 25. 0 aplicado a las ciencias sociales. Para contrastar las hipótesis planteadas se empleó la prueba de normalidad de Kolmogórov - Smirnov. Se utilizó la prueba estadística Chi-cuadrada para determinar si existe asociación directa, estrecha y significative entre las variables estudiadas.

\section{Resultados}

\section{Niveles de funcionamiento familiar}

\section{Tabla 1}

Niveles de funcionamiento familiar

\begin{tabular}{|c|c|c|c|c|c|c|c|c|c|}
\hline & & \multicolumn{2}{|c|}{ Muy bajo } & \multicolumn{2}{|r|}{ Bajo } & \multicolumn{2}{|r|}{ Alto } & \multicolumn{2}{|c|}{ Muy } \\
\hline & & $\mathrm{n}$ & $\%$ & $\mathrm{n}$ & $\%$ & $\mathrm{n}$ & $\%$ & $\mathrm{n}$ & $\%$ \\
\hline \multirow{2}{*}{$\begin{array}{l}\text { Funcionamiento } \\
\text { familiar }\end{array}$} & Cohesión & 68 & $11.3 \%$ & 256 & $42.5 \%$ & 217 & $36.0 \%$ & 62 & $10.3 \%$ \\
\hline & Adaptabilidad & 28 & $4.6 \%$ & 215 & $35.7 \%$ & 246 & $40.8 \%$ & 114 & $18.9 \%$ \\
\hline
\end{tabular}

En la tabla 1 se observa que el $42.5 \%$ de estudiantes presentan un nivel bajo de cohesión familiar, mientras que, el 36 \% muestra niveles altos de cohesión familiar. Respecto a la dimensión adaptabilidad familiar el $40.8 \%$ muestra un alto nivel, por el contrario, el 35. $7 \%$ evidencia un nivel bajo.

\section{Niveles de adicción a redes sociales}

\section{Tabla 2}

Niveles de adicción a redes sociales

\begin{tabular}{|c|c|c|c|c|c|c|c|c|}
\hline & \multicolumn{2}{|c|}{ Riesgo bajo } & \multicolumn{2}{|c|}{ Riesgo medio } & \multicolumn{2}{|c|}{ Riesgo alto } & \multicolumn{2}{|c|}{ Adicción } \\
\hline & $\mathrm{n}$ & $\%$ & $\mathrm{n}$ & $\%$ & $\mathrm{n}$ & $\%$ & $\mathrm{n}$ & $\%$ \\
\hline Adicción a redes sociales Global & 301 & $49.9 \%$ & 216 & $35.8 \%$ & 71 & $11.8 \%$ & 15 & $2.5 \%$ \\
\hline Pérdida del control & 257 & $42.6 \%$ & 241 & $40.0 \%$ & 86 & $14.3 \%$ & 19 & $3.2 \%$ \\
\hline Síndrome de abstinencia & 241 & $40.0 \%$ & 282 & $46.8 \%$ & 71 & $11.8 \%$ & 9 & $1.5 \%$ \\
\hline Disminución del rendimiento académico & 340 & $56.4 \%$ & 208 & $34.5 \%$ & 50 & $8.3 \%$ & 5 & $0.8 \%$ \\
\hline Modificación del estado de ánimo & 373 & $61.9 \%$ & 151 & $25.0 \%$ & 60 & $10.0 \%$ & 19 & $3.2 \%$ \\
\hline Dependencia & 440 & $73.0 \%$ & 109 & $18.1 \%$ & 41 & $6.8 \%$ & 13 & $2.2 \%$ \\
\hline Pérdida de interés por otras actividades & 330 & $54.7 \%$ & 196 & $32.5 \%$ & 67 & $11.1 \%$ & 10 & $1.7 \%$ \\
\hline Interferencia en el área social & 367 & $60.9 \%$ & 176 & $29.2 \%$ & 49 & $8.1 \%$ & 11 & $1.8 \%$ \\
\hline
\end{tabular}


En la tabla 2 se aprecia que el $49.9 \%$ de estudiantes muestran un nivel de riesgo bajo de adicción a redes sociales, mientras que, el $35.8 \%$ evidencia un riesgo medio, por otro lado, el $11.8 \%$ se encuentra en riesgo alto y el $2.5 \%$ presenta una franca adicción. En cuanto a las dimensiones, se evidencia que la mayoría de estudiantes muestran un riesgo bajo en todas las dimensiones: pérdida del control (42.6\%), síndrome de abstinencia (40,2\%), disminución del rendimiento académico (56.4\%), modificación del estado de ánimo (61,9\%), dependencia (73\%), pérdida de interés por otras actividades $(54.7 \%)$ e interferencia en el área social (60.9 $\%)$.

\section{Prueba de normalidad de dos variables}

Tabla 3

Prueba de bondad de ajuste para las variables de estudio

\begin{tabular}{|c|c|c|c|c|c|}
\hline Instrumento & Variable & $\begin{array}{l}\text { Medi } \\
\mathrm{a}\end{array}$ & D.E & $\begin{array}{l}\text { K- } \\
\text { S } \\
\end{array}$ & $\mathrm{P}$ \\
\hline \multirow{4}{*}{ Funcionamiento familiar } & Cohesión & 36.9 & 5.96 & 1.07 & 0.20 \\
\hline & & 8 & 6 & 2 & 1 \\
\hline & Adaptabilidad & 30.1 & 5.51 & 1.72 & 0.00 \\
\hline & & 2 & 9 & 7 & 5 \\
\hline \multirow{16}{*}{ Adicción a redes sociales } & Adicción global & 48.1 & 32.95 & 1.76 & 0.00 \\
\hline & & 4 & 7 & 9 & 4 \\
\hline & Pérdida del control & 13.0 & 8.63 & 1.76 & 0.00 \\
\hline & & 9 & 7 & 7 & 4 \\
\hline & Síndrome de abstinencia & 9.41 & 5.68 & 1.58 & 0.01 \\
\hline & & & 9 & 1 & 4 \\
\hline & Disminución del rendimiento académico & 4.47 & 3.69 & 2.82 & .00 \\
\hline & & 5.00 & 5 & 2 & 0 \\
\hline & Modificación del estado de ánimo & & 4.59 & 3.42 & .00 \\
\hline & & & 0 & 5 & 0 \\
\hline & Dependencia & 3.75 & 4.29 & 4.76 & .00 \\
\hline & & & 0 & 7 & 0 \\
\hline & Pérdida de interés por otras actividades & 7.56 & 6.16 & 2.70 & .00 \\
\hline & & & 9 & 9 & 0 \\
\hline & Conflicto en el ambiente familiar & 4.86 & 4.15 & 3.16 & .00 \\
\hline & & & 8 & 7 & 0 \\
\hline
\end{tabular}

En la tabla 3 se muestra la prueba de bondad de ajuste con la finalidad de contrastar las hipótesis planteadas, para definir si las variables presentan una distribución normal, se utilizó la prueba de bondad de ajuste de Kolmogórov - Smirnov (K-S), dando como resultado una distribución no normal de las variables, debido a que, el coeficiente obtenido no es significativo ( $\mathrm{p}<0.05)$. Razón por la cual, se utilizó Chi-cuadrada para encontrar el nivel de asociación entre las variables. 


\section{Análisis de asociación entre las variables de estudio}

\section{Tabla 4}

Niveles de asociación entre las dimensiones del funcionamiento familiar y adición a redes sociales

Adicción a redes sociales

\begin{tabular}{|c|c|c|c|c|c|c|c|c|c|}
\hline & & \multicolumn{2}{|c|}{ Riesgo bajo } & \multicolumn{2}{|c|}{ Riesgo medio } & \multicolumn{2}{|c|}{ Riesgo alto } & \multicolumn{2}{|c|}{ Adicción } \\
\hline & & $\mathrm{n}$ & $\%$ & $\mathrm{n}$ & $\%$ & $\mathrm{n}$ & $\%$ & $\mathrm{n}$ & $\%$ \\
\hline \multirow{5}{*}{ Cohesión } & Muy bajo & 30 & 10 & 27 & 12,5 & 10 & 12,1 & 1 & 6,7 \\
\hline & Bajo & 105 & 34,9 & 112 & 51,9 & 35 & 49,3 & 4 & 26,7 \\
\hline & Alto & 120 & 39,9 & 68 & 31,5 & 22 & 31 & 7 & 46,7 \\
\hline & Muy alto & 46 & 15,3 & 9 & 4,2 & 4 & 5,6 & 3 & 20 \\
\hline & Muy bajo & 19 & 6,3 & 9 & 4,2 & 0 & 0 & 0 & 0 \\
\hline \multirow{3}{*}{ Adaptabilidad } & Bajo & 108 & 35,9 & 80 & 37 & 26 & 36,6 & 1 & 6,7 \\
\hline & Alto & 119 & 39,5 & 97 & 44,9 & 27 & 38 & 3 & 20 \\
\hline & Muy alto & 55 & 18,3 & 30 & 13,9 & 18 & 25,4 & 11 & 73,3 \\
\hline
\end{tabular}

En la tabla 4 se muestra que el mayor porcentaje de estudiantes presentan niveles altos de cohesión y adaptabilidad familiar (39.9 \% y $39.5 \%$ ), de igual manera, se evidencia que este mismo grupo presenta bajos niveles de adición a redes sociales, lo cual significa que, existe una asociación significativa entre funcionamiento familiar y adicción a redes sociales.

\section{Análisis de asociación entre las dimensiones del funcionamiento familiar y adicción a redes sociales}

\section{Tabla 5}

Análisis de asociación entre las dimensiones del funcionamiento familiar y adicción a redes sociales

\begin{tabular}{|c|c|c|c|c|}
\hline & & Valor & $\mathrm{gl}$ & $\mathrm{p}$ \\
\hline Cohesión & Chi-cuadrado de Pearson & $\begin{array}{c}33,236 \\
\mathrm{a}\end{array}$ & 9 & ,000 \\
\hline Adaptabilidad & Chi-cuadrado de Pearson & $\begin{array}{c}40,318 \\
\mathrm{a}\end{array}$ & 9 & 000 \\
\hline
\end{tabular}

La tabla 5 revela que existe asociación entre el funcionamiento familiar y adicción a redes sociales. Asimismo, se encontró asociación altamente significativa entre cohesión 
familiar y adición a redes sociales $\left(\mathrm{X}^{2}=33,236 ; \mathrm{p}=, 000\right)$. De la misma manera, muestra una asociación altamente significativa entre adaptabilidad familiar y adicción a redes sociales $\left(X^{2}=40,318 ; p=, 000\right)$. Lo que significa que, el funcionamiento familiar se asocia directamente con la adicción a redes sociales, es decir, mientras mayor sea el nivel de cohesión y adaptabilidad familiar, menores serán los riesgos de una adicción a redes sociales.

\section{Discusión}

El propósito de esta investigación fue determinar si existe asociación entre el funcionamiento familiar (según las dimensiones de cohesión y adaptabilidad) y adicción a redes sociales en estudiantes del nivel secundario de una institución educativa pública de Lima este. Los resultados encontrados en esta investigación evidencian que existe una asociación altamente significativa entre cohesión familiar y adicción a redes sociales $\left(\mathrm{X}^{2}=\right.$ 33, 236; $\mathrm{p}=$,000), es decir, los estudiantes que presentan mejores niveles de cohesión familiar, mostrarán también menor riesgo de padecer una adicción a redes sociales. Estos resultados coinciden con los encontrados por Adriano y Mamani (2015) quienes hallaron una asociación altamente significativa e inversa $(r=-, 223 ; \mathrm{p}<0,01)$ entre cohesión familiar y adicción a redes sociales, esto significa que, al existir mayor cohesión familiar, la familia tendrá la capacidad de afrontar diferentes situaciones estresantes de manera conjunta, es por ese motivo que, el vínculo emocional y afectivo que existe entre los miembros de la familia es de suma importancia, ya que permite lograr una relación familiar más estrecha y, para fortalecer ese vínculo es necesario compartir tiempo, actividades, decisiones y afectos como familia, para así de esa manera, lograr un sistema familiar optimo que forme niños y adolescentes que puedan ser capaces de darse cuenta y limitarse ante una adicción, pero sobre todo a una muy sutil como lo es las redes sociales.

Del mismo modo, Haro (2018) encontró una relación altamente significativa y negativa $\left(\mathrm{r}=-, 235^{* *} ; \mathrm{p}<0,01\right)$ entre cohesión familiar y adicción a redes sociales en adolescentes de 12 a 17 años, lo que significa que, las familias con niveles altos de cohesión familiar se caracterizarán principalmente por mantener una relación estrecha, con un adecuado vínculo emocional entre sus miembros, por eso, quienes han sido formados en este tipo de familias, serán capaces de controlar el acceso y uso de las redes sociales, o, por lo contrario, de presentarse una adicción en alguno de sus miembros, la familia mostrará

preocupación y brindará su apoyo para superar la situación problemática, siendo esta, la fuente principal de soporte psicológico. Asimismo, Pinto (2018) encontró relación altamente significativa (rho $=-10^{* *}, \mathrm{p}=, 000$ ) entre el funcionamiento familiar y la adicción a redes sociales, mostrando resultados similares con esta investigación. 
En relación a adaptabilidad familiar y adicción a redes sociales, se halló asociación altamente significativa $\left(\mathrm{X}^{2}=40,318 ; \mathrm{p}=, 000\right)$, dicho en otras palabras, los estudiantes con familias con altos niveles de adaptabilidad familiar, presentarán menor riesgo de una adicción a redes sociales. Estos datos, se pueden comprender de mejor manera en la investigación realizada por Martínez (2015) quien menciona que bajos niveles de adaptabilidad se relacionan de manera positiva con las adicciones que indica que niveles muy altos o muy bajos de adaptabilidad familiar pueden conformar sistemas caóticos donde las normas de funcionamiento no están claras, ni existen límites establecidos, llevando al adolescentes muchas veces a afrontar situaciones de riesgo que pueden desencadenarse en una adicción a redes sociales. Del mismo modo, Pinto (2018) encontró relación altamente significativa (rho $\left.=-, 14^{* *} ; \mathrm{p}=, 000\right)$ entre adaptabilidad familiar y adicción a redes sociales, esto significa que, un desbalance en el sistema familiar producido por conductas extremas de sus miembros, provocaría mayor probabilidad de que alguno de ellos presente una adicción a redes sociales, aunque, la familia siempre tendrá la opción de modificar su estructura, comportamiento y decisiones con el propósito de enfrentar cualquier cambio en sus relaciones o en situaciones adversas. De igual manera, Martínez (2015) menciona que los sistemas familiares necesitan tanto la estabilidad como el cambio, ya que, estas evolucionan y se desarrollan, es por esta razón, que la capacidad de cambiar es una de las característica que diferencia a las familias funcionales de las disfuncionales.

Estos resultados discrepan con los encontrados por Adriano y Mamani (2015) quienes no hallaron relación significativa $(r=-, 112 ; \mathrm{p}>0,05)$ entre estas variables, debido a que, esta dimensión posee libertad para cambiar su estructura de poder, sus roles y reglas de relación entre sus miembros en respuesta a situaciones estresantes o evolutivas. De la misma forma, Haro (2018) no encontró relación entre las variables $(\mathrm{r}=, 128 ; \mathrm{p}<0,01)$, esto se debe principalmente a múltiples factores que intervienen en una adicción a redes sociales, como rasgos de personalidad (introversión, timidez o sentimientos de inferioridad), baja autoestima y alteraciones psicopatológicas (adicciones químicas o psicológicas ideación e intento de suicidio o depresión) que incrementan el riesgo de adicción a redes sociales.

Los resultados finalmente sostendrían que los adolescentes con familias cohesionadas y adaptables en niveles altos, mostrarán menor riesgo de presentar una adicción a redes sociales, aunque se sabe que durante esta etapa, los adolescentes, tienden a ser más vulnerables, porque existe mayor probabilidad de que adquieran alguna conducta adictiva, ya que estos, suelen realizar actividades e intereses fuera de la familia, centrándose en su grupo de amigos, cediendo muchas veces a la presión social para encajar en el grupo, a esto se le suma las recompensas inmediatas que ofrecen las redes sociales. Finalmente, se debe tener 
en cuenta lo que indican Cruz et al. (2011) que una familia con un buen funcionamiento debe crear un ambiente saludable para los niños y adolescentes, un lugar en donde se posea la capacidad de cambiar y los límites claros deben ser establecidos desde los primeros años de vida del menor.

\section{Conclusiones}

En relación al objetivo general, se evidencia que existe asociación altamente significativa entre el funcionamiento familiar y adicción a redes sociales en estudiantes del nivel secundario de una institución educativa pública de Lima Este. En cuanto al primer objetivo específico, se encontró asociación altamente significativa entre cohesión familiar y adicción a redes sociales en estudiantes del nivel secundario de una institución educativa pública de Lima Este. Respecto al segundo objetivo específico, se encontró asociación altamente significativa entre adaptabilidad familiar y adicción a redes sociales en estudiantes del nivel secundario de una institución educativa pública de Lima Este.

Al finalizar esta investigación, se recomienda lo siguiente: Realizar investigaciones con muestras más amplias, que permitan comparar las variables estudiadas de acuerdo a su género, condición social o cultural. Tomar en cuenta otras variables de estudio que influyan en el funcionamiento familiar o en la adicción a redes sociales como la personalidad, habilidades sociales, depresión, autoestima e inteligencia emocional. Promover activamente en las escuelas el desarrollo de actividades para el uso responsable de las redes sociales en los adolescentes. Sensibilizar a la comunidad educativa sobre la importancia de la familia para la prevención de adicciones. Compartir tiempo y actividades familiares para fortalecer el vínculo afectivo y emocional entre sus miembros.

\section{Referencias}

Adriano, C., \& Mamani, L. (2015). Funcionamiento familiar y adicción a Internet en estudiantes de una institución educativa pública de Lima norte-2014 [Tesis de licenciatura, Universidad Unión]. https://repositorio.upeu.edu.pe/bitstream/handle/UPEU/235/Luis_Tesis_bachiller_2 015.pdf? sequence $=1 \&$ is Allowed $=\mathrm{y}$

Aguirre, M., \& Zurita, M. (2015). Ciberadicción y funcionalidad familiar en adolescentes de octavo de básica y tercer año de bachillerato en el colegio militar Miguel Iturralde de Portoviejo y colegio militar Eloy Alfaro de Quito en el mes de enero de 2015 [Tesis de licenciatura, Pontificia Universidad Católica de Quito]. http://repositorio.puce.edu.ec/handle/22000/8874 
Ángel, M., \& Álzate, Y. (2015). Relaciones familiares y sociales en adolescentes usuarios de redes sociales virtuales. Revista de Ciencias Sociales, 20. https://dialnet.unirioja.es/servlet/articulo?codigo $=5585568$

Aponte, D., Castillo, P., \& González, J. (2017). Prevalencia de adicción a internet y su relación con disfunción familiar en adolescentes. Revista Clínica de Medicina de Familia, 10 (3), 179-186. http://scielo.isciii.es/pdf/albacete/v10n3/1699-695X-albacete-10-03-179.pdf

Bazo, J., Bazo, O., Águila, J., Peralta, F., Mormontoy, W., \& Bennett, I. (2017). El modelo circumplejo tridimensional (3 - D): Sobre las mediciones no - curvilíneas del FACES III. Revista Peruana de Medicina Experimental y Salud Publica, 34 (1), 151-152. Doi:10.17843/rpmesp.2017.341.2782

Castro, C. (2018). Riesgo de adicción a redes sociales y comunicación familiar en adolescentes de una Institución Educativa [Tesis de maestria, Universidad Peruana Unión]. https://repositorio.upeu.edu.pe/bitstream/handle/UPEU/2913/Carlos_Tesis_Maestro _2019.pdf? sequence $=4 \&$ isAllowed $=\mathrm{y}$

Córdova, E., \& Dávila, G. (2017). Clima social familiar y adicción a las redes sociales en los estudiantes del colegio militar Andrés Avelino Cáceres de Morales - San Martín, 2017 [Tesis de licenciatura, Universidad Peruana Unión - Filial Tarapoto]. https://repositorio.upeu.edu.pe/bitstream/handle/UPEU/1441/Elizabeth_Tesis_T\%c3 \%adtulo_2018.pdf?sequence $=3 \&$ is Allowed $=\mathrm{y}$

Cruzado, L., Matos, L., y Kendall, R. (2006). Adicción a internet: perfil clínico y epidemiológico de pacientes hospitalizados en un instituto nacional de salud mental. Revista Médica Herediana, 17 (4), 196-205. http://www.scielo.org.pe/pdf/rmh/v17n4/v17n4ao2.pdf

Echeburúa, E., Labrador, F., \& Becoña, E. (2009). Adicción a las nuevas tecnologías en adolescentes y jóvenes. Madrid, España: Pirámide.

Echeburúa, E. (2009). Redes sociales: la nueva adicción a internet. España: Pirámide.

Echeburúa, E., \& Corral, P. (2010). Adicción a las nuevas tecnologías y a las redes sociales en jóvenes: un nuevo reto. Adicciones, 22 (2), 91-96. http://www.adicciones.es/index.php/adicciones/article/view/196/186

Gavilanes, G. (2015). Adicción a redes sociales y su relación con la adaptación conductual en los adolescentes [Tesis de licenciatura, Pontificia Universidad Católica del Ecuador]. https://repositorio.pucesa.edu.ec/bitstream/123456789/1137/1/75670.pdf 
Haro, E. (2018). Adicción a redes sociales y funcionalidad familiar en estudiantes universitarios y egresados de Lima Metropolitana [Tesis de licenciatura, Universidad San Ignacio de Loyola]. http://repositorio.usil.edu.pe/bitstream/USIL/3684/3/2018_Haro-Arrarte.pdf.pdf

Hernández, R., Fernández, C., \& Baptista, P. (2005). Metodología de la investigación. México: McGraw-Hill Interamericana.

Maldonado, E. (2015). Las redes sociales y la convivencia familiar de los estudiantes de quinto a noveno año de educación básica del centro educativo bilingüe Thomas Cranmer de la ciudad de Ambato [Tesis de licenciatura, Universidad Técnica de Ambato]. https://repositorio.uta.edu.ec/bitstream/123456789/9912/1/FJCS-TS171.pdf

Martínez, J. (2015). Funcionamiento familiar y apoyo social en el consumo de drogas y las conductas adictivas de los adolescentes [Tesis doctoral, Universidad de Málaga]. https://riuma.uma.es/xmlui/bitstream/handle/10630/10089/TD_Martinez_Peralta.pdf ?sequence $=1$

Morales, L. (2013). Factores de riesgo asociados a la conducta de adicción a internet en adolescentes de la I.E. Jorge Martorell Flores, Tacna-2012 [Tesis de licenciatura, Universidad Nacional Jorge Basadre Grohmann]. http://repositorio.unjbg.edu.pe/bitstream/handle/UNJBG/467/TG0324.pdf?sequence $=1 \&$ is Allowed $=\mathrm{y}$

Navarro, A., \& Rueda, G. (2007). Adicción a Internet: revisión crítica a la literatura. Revista

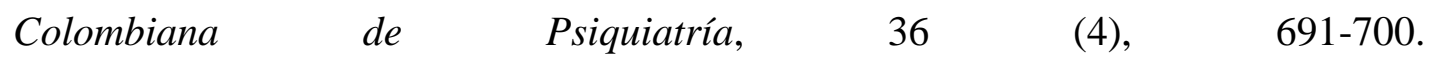
https://www.redalyc.org/pdf/806/80636409.pdf

Oliva, C. (2012). Redes sociales y jóvenes: una intimidad cuestionada en internet. Revista de Ciencias sociales $\quad$ Aposta, $\quad 3 \quad$ 1-16. https://www.redalyc.org/pdf/4959/495950250003.pdf

Olson, D., Sprenkle, D., \& Russell, C. (1979). Circumplex model of marital and family system: I. Cohesion and adaptability dimensions, family types, and clinical $\begin{array}{lllll}\text { applications. } & \text { Family } & \text { Process, } & 18 & \text { (1), }\end{array}$ https://www.ncbi.nlm.nih.gov/pubmed/437067

Pinto, A. (2018). Funcionamiento familiar y adicción a las redes sociales en estudiantes de tercero, cuarto y quinto de secundaria [Tesis de licenciatura, Universidad Nacional Mayor de San Marcos]. https://cybertesis.unmsm.edu.pe/bitstream/handle/20.500.12672/9785/Pinto_ba.pdf? sequence $=1 \&$ is Allowed $=\mathrm{y}$ 
Rayo, A. (2014). Influencia del uso de las redes sociales en las relaciones familiares de jóvenes de 18 y 24 años que presentan adicción a las mismas [Tesis de licenciatura, Universidad Rafael Landívar]. http://biblio3.url.edu.gt/Tesario/2014/05/42/RayoAlejandra.pdf

Sammarco, P. (2019). Descubre todo sobre las redes sociales, The social media family. https://thesocialmediafamily.com/redes-sociales/

Sánchez, H., Reyes, C., \& Mejía, K. (2018). Manual de términos e investigación científica, tecnología y humanística. Lima: Editorial de la Universidad Ricardo Palma. http://repositorio.urp.edu.pe/handle/URP/1480

Sigüenza, W., Buñay, R., \& Guamán, M. (2017). Funcionamiento familiar real e ideal según modelo circumplejo de Olson. Maskana, 8, 77-85.

https://publicaciones.ucuenca.edu.ec/ojs/index.php/maskana/article/view/1878/1378

Urquijo, J. (2017). Sociedad y nuevas tecnologías, ventajas e inconvenientes. Revista extremena de ciencias sociales Almenara, 1 (9), 45-49.

https://dialnet.unirioja.es/servlet/articulo?codigo $=5889948$

Vallejos, M., \& Capa, W. (2010). Videojuegos: adicción y factores predictores. Avances, $18(1), \quad \quad 103-110$.

https://www.academia.edu/36905106/VIDEO_JUEGOS_ADICCI\%C3\%93N_Y_F

A CTORES_PREDICTORES_Video_Games_Addiction_and_predictors

Vilca, L., \& Vallejos, M. (2015). Construcción of the Risk of Addiction to Social Networks Scale (CrARS). Computers in Human Behavior (48), 190-198. Recuperado de https://dl.acm.org/citation.cfm?id=2781900.2782111

We are social, y Hootsuits. (2019). Digital 2019: essential Insight into how people around the worl use internet, movil device, media social, and e-commerce. https://wearesocial.com/global-digital-report-2019

Zaldívar, D. (2012). Funcionamiento familiar saludable. Revista de salud cubana, 1-4. http://www.sld.cu/galerias/pdf/sitios/rehabilitaciontemprana/funcionamiento_famili a r.pdf

Zambrano, A. (2011). Cohesión, adaptabilidad familiar y el rendimiento académico en comunicación de alumnos de una institución educativa del Callao [Tesis de maestría, Universidad San Ignacio de Loyola]. https://repositorio.usil.edu.pe/handle/123456789/1305

Zapata, G. (2013). Uso problemático de internet en adolescentes atendidos en consulta externa del Hospital Hermilio Valdizán en el periodo 2009-2011 [Tesis de maestría, Universidad Nacional Mayor de San Marcos]. 
http://ateneo.unmsm.edu.pe/bitstream/handle/123456789/2971/Zapata_Coacalla_Gi

ovanna_2013.pdf? sequence $=1 \&$ isAllowed $=\mathrm{y}$ 